\title{
Low-energy electron scattering by silane $\left(\mathrm{SiH}_{4}\right)$
}

\author{
C. Winstead and V. McKoy \\ A. A. Noyes Laboratory of Chemical Physics, California Institute of Technology, Pasadena, California 91125 \\ (Received 11 December 1989; revised manuscript received 9 July 1990)
}

\begin{abstract}
We have applied the Schwinger multichannel formulation [K. Takatsuka and V. McKoy, Phys. Rev. A 24, 2473 (1981)] to the elastic scattering of low-energy (1-30-eV) electrons by silane. Our results, obtained within the fixed-nuclei, static-exchange approximation, are in generally good agreement with recent differential, integral, and momentum-transfer cross-section measurements between 5 and $30 \mathrm{eV}$. At lower energies, our results reflect the known limitations of the static-exchange approximation. Near the shape resonance, the calculated differential cross section shows significant enhancement at high angles, suggesting an explanation for differences between integral cross sections determined from integrated differential data and those obtained from transmission measurements.
\end{abstract}

\section{INTRODUCTION}

The scattering of electrons by silane is an important process in low-temperature plasmas used for semiconductor processing and fabrication. ${ }^{1} \mathrm{SiH}_{4}$ is also of theoretical interest as an analog of $\mathrm{CH}_{4}$, which has served as a prototypical polyatomic molecule for electron-scattering calculations. There have been several experimental or experimentally based studies of $e^{-}-\mathrm{SiH}_{4}$ collisions, ${ }^{2-11}$ and the general features of the elastic and momentum-transfer cross sections are well known. Like methane, silane exhibits both a Ramsauer-Townsend minimum ${ }^{2}$ and a broad shape resonance. ${ }^{3-7}$ Recently, Tanaka and coworkers ${ }^{6}$ have reported differential elastic and vibrational excitation cross sections, while Tronc, Hitchcock, and Edard $^{11}$ have measured fixed-angle vibrational and valence electronic excitation functions. Within the past six years, numerous theoretical studies of $e^{-}-\mathrm{SiH}_{4}$ scattering have appeared, beginning with the multiplescattering $X \alpha$ calculation of Tossell and Davenport, ${ }^{12}$ which showed a $d$-wave resonance, ascribed to $T_{2}$ symmetry, in the total elastic cross section, but which gave a cross section much too large in comparison to subsequent experiments. Jain and co-workers; ${ }^{13-15}$ Gianturco, Pantano, and Scialla; ${ }^{16}$ and Yuan ${ }^{17,18}$ have studied electronsilane collisions using a variety of approximate treatments of exchange and polarization. Good agreement with experiment ${ }^{4}$ is obtained above $50 \mathrm{eV} .{ }^{13}$ Some of these theoretical studies ${ }^{13-15,18}$ report momentumtransfer cross sections, but the only calculated differential cross sections which have been published are those of Jain ${ }^{13}$ above $30 \mathrm{eV}$ and the $X \alpha$ results of Tanaka and coworkers ${ }^{6}$ from 1.8 to $100 \mathrm{eV}$. The latter reproduce the shape of the experimental cross sections but are consistently lower than experiment.

Here we have used the Schwinger multichannel ${ }^{19,20}$ (SMC) procedure to study low-energy elastic electron scattering by $\mathrm{SiH}_{4}$ within the fixed-nuclei, static-exchange approximation. We report elastic and momentumtransfer cross sections from 1 to $30 \mathrm{eV}$. The present calculation, which treats exchange correctly but neglects po- larization entirely, is intended to serve as a reference point for planned SMC studies of elastic $e^{-}-\mathrm{SiH}_{4}$ scattering incorporating polarization, and for studies of electronic excitation which will be carried out at a level comparable to static exchange, that is, retaining a small number of open channels and generally neglecting closed channels. The present calculation also provides an indication of the reliability of static-exchange calculations which are currently under way for larger polyatomic systems such as disilane, tetrafluorosilane, and germane. More sophisticated theoretical treatments of these larger molecules can be quite demanding, and experimental data are limited. It is therefore important to understand the range of usefulness of the static-exchange approximation as applied to polyatomic systems. In the present study of $\mathrm{SiH}_{4}$, the agreement of our differential cross sections with the recent measurements of Tanaka and co-workers ${ }^{6}$ is good above $4 \mathrm{eV}$. Our integral and momentum-transfer cross sections also agree well with experiment over this range, with certain exceptions noted below. From 1 to 4 $\mathrm{eV}$ the static-exchange approximation expectedly does less well, ${ }^{21-23}$ due to omission of polarization.

The remainder of this paper is organized into three sections. Section II gives some relevant theoretical and computational details. In Sec. III we present our results and compare them to experiment and to other calculations. Section IV contains a brief discussion and concluding remarks.

\section{THEORY}

We have used the Schwinger multichannel formulation of McKoy and co-workers, ${ }^{19,20}$ which for the elastic, static-exchange problem considered here is equivalent to the original Schwinger variational method. The references cited contain a thorough discussion of our procedure, so we confine ourselves here to a summary of the relevant features. In the SMC procedure, the $(N+1)$ electron wave function $\Psi_{N+1}(E)$ of the electron-molecule system is expanded in a basis of Slater determinants of Gaussian molecular orbitals, and the coefficients of this 
expansion are determined by requiring that the Schwinger functional for the $T$ matrix be stationary with respect to their variation. Exchange is correctly accounted for by the antisymmetrization of $\Psi_{N+1}(E)$. In the present calculation, only determinants arising from the addition of one electron to the SCF ground-state wave function of $\mathrm{SiH}_{4}$ are included in the expansion of $\Psi_{N+1}(E)$. Polarization effects can be accounted for by inclusion of energetically closed channels in the expansion of the scattering wave function. ${ }^{22-25}$ Staticexchange calculations such as the present one have yielded generally reliable results above $5 \mathrm{eV} .^{21,22,26,27}$

The principal factor affecting the quality of these elastic-scattering calculations is the nature of the $L^{2}$ basis used to represent the target and scattering orbitals. In our study of $\mathrm{SiH}_{4}$ we have employed three basis sets. The first, a very restricted set used only for exploratory calculations, consists of the $(12 s, 8 p) /[4 s, 2 p]$ set of McClean and Chandler ${ }^{28}$ for Si, supplemented by two $d$ Cartesian Gaussians with exponents 0.2 and 0.07 , respectively, and the $(4 s) /[3 s]$ set of Pacansky and Dupuis ${ }^{29}$ and Van Duijneveldt $^{29,30}$ for $\mathrm{H}$. All of the results presented below were obtained in a somewhat larger basis, consisting of the above basis supplemented by two $s$ orbitals, with exponents 0.05 and 0.02 , and two $p$ orbitals, with exponents 1.0 and 0.05 , located on the silicon center. The results obtained in the larger basis have been checked against calculations in a third basis containing an additional $s, p$, and $d$ Cartesian Gaussian on the $\mathrm{Si}$ center (exponents $0.007,0.02$, and 0.025 , respectively), and appear converged to approximately $1 \%$. Although these Gaussian basis sets are modest in comparison to those typically used in earlier SMC calculations, ${ }^{21-27}$ in those earlier studies the choice of basis was largely driven by the need to achieve approximate completeness in the basis-set expansion of the free-particle Green's function occurring in the $V G_{P}^{(+)} V$ matrix elements. We have recently implemented a numerical procedure ${ }^{31}$ for evaluating these terms that eliminates the need for such expansions. The improved procedure permits the choice of basis to be governed by the dynamics of the low-energy scattering process, considerably reducing the basis size required. In tests on elastic scattering by $\mathrm{CH}_{4}$, we found that quite small basis sets could give excellent results. ${ }^{31,32}$

\section{RESULTS}

In Fig. 1 we show our total elastic cross section in comparison with the available experimental data $^{5-7}$ and with selected calculated results. ${ }^{14,16}$ Tabulated values are available on request. The magnitude of the staticexchange cross section is seen to agree well with experiment over this energy range. Our calculation shows a resonance maximum at $5 \mathrm{eV}$, which is a somewhat lower energy than reported by Tanaka and co-workers, ${ }^{6}$ but higher than found by Sueoka and co-workers ${ }^{5}$ or Wan, Moore, and Tossell. ${ }^{7}$ Since neglect of polarization in the present work should put the resonance in our cross section at somewhat higher energies than is actually the case, our results tend to support the data of Refs. 5 and 7 over those of Ref. 6 . Earlier calculations ${ }^{14-18}$ that in-

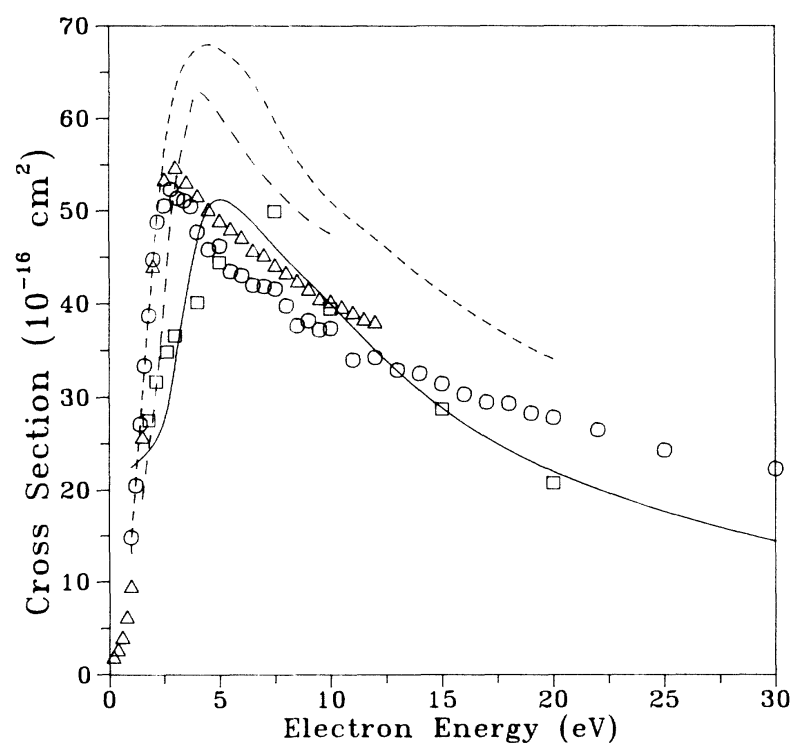

FIG. 1. Total elastic cross section for scattering of electrons by $\mathrm{SiH}_{4}$. Theoretical results shown are the present work (solid line), the "JT" results of Ref. 14 (long dashes), and the "MSCEP" results of Ref. 16 (short dashes). Experimental values are from Refs. 5 (circles), 6 (squares), and 7 (triangles).

cluded approximate treatments of polarization tend to place the resonance at about $4 \mathrm{eV}$. Above $10 \mathrm{eV}$, our results agree best with the data of Ref. 6; however, at these energies the total cross sections of Refs. 5 and 7 may include contributions from electronically inelastic scattering. While we have not continued our calculations below $1 \mathrm{eV}$, there is no indication of a Ramsauer-Townsend

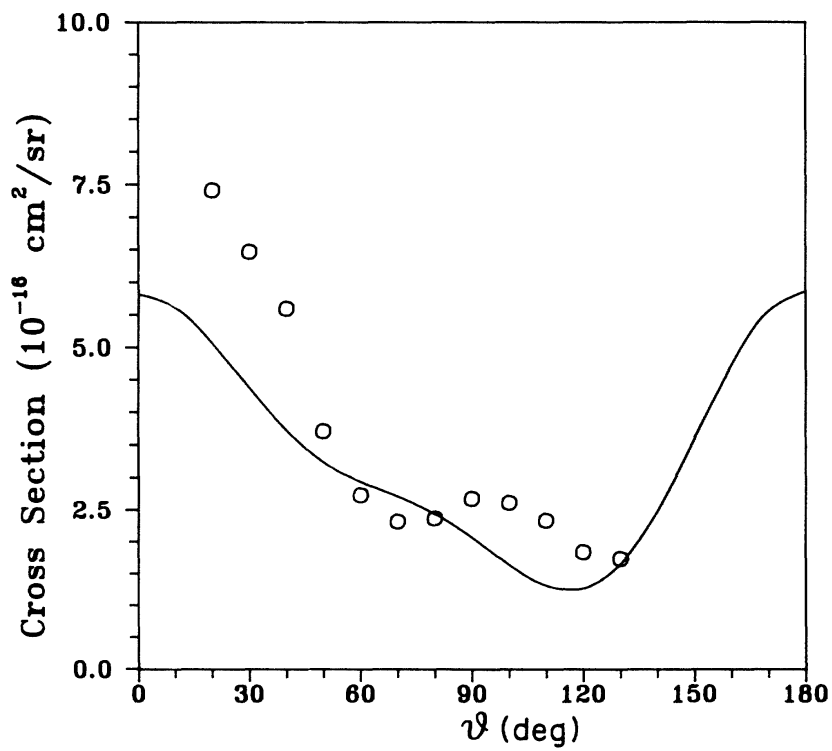

FIG. 2. Differential cross section for elastic scattering of 3$\mathrm{eV}$ electrons by $\mathrm{SiH}_{4}$. The solid curve is the result of the present calculation; the circles are the experimental values of Ref. 6. 


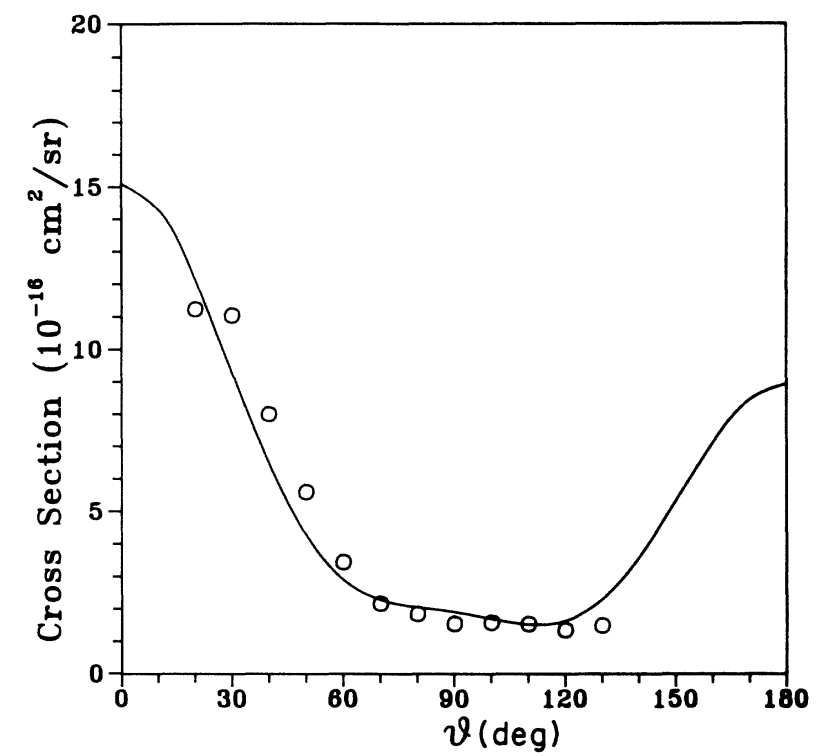

FIG. 3. Same as in Fig. 2, at $4 \mathrm{eV}$.

minimum in the trend of the static-exchange cross section. This is not surprising, since earlier studies ${ }^{21,22}$ explicitly showed that the Ramsauer-Townsend minimum in $e^{-}-\mathrm{CH}_{4}$ scattering is not seen at the static-exchange level.

Our results for the differential cross section are shown at selected energies in Figs. 2-8, along with the measurements of Ref. 6. At low energies, the $d$-wave character of the resonance, already identified by Tossell and Davenport, ${ }^{12}$ is quite apparent in the form of both the experimental and the theoretical cross sections, although the two do not agree in detail. Again, such differences are to

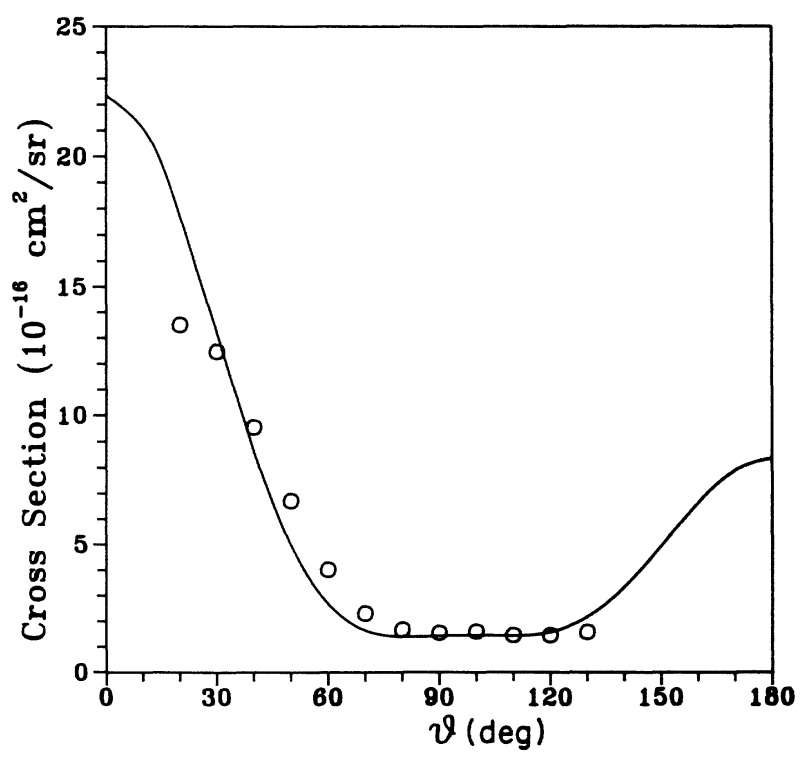

FIG. 4. Same as in Fig. 2, at $5 \mathrm{eV}$.

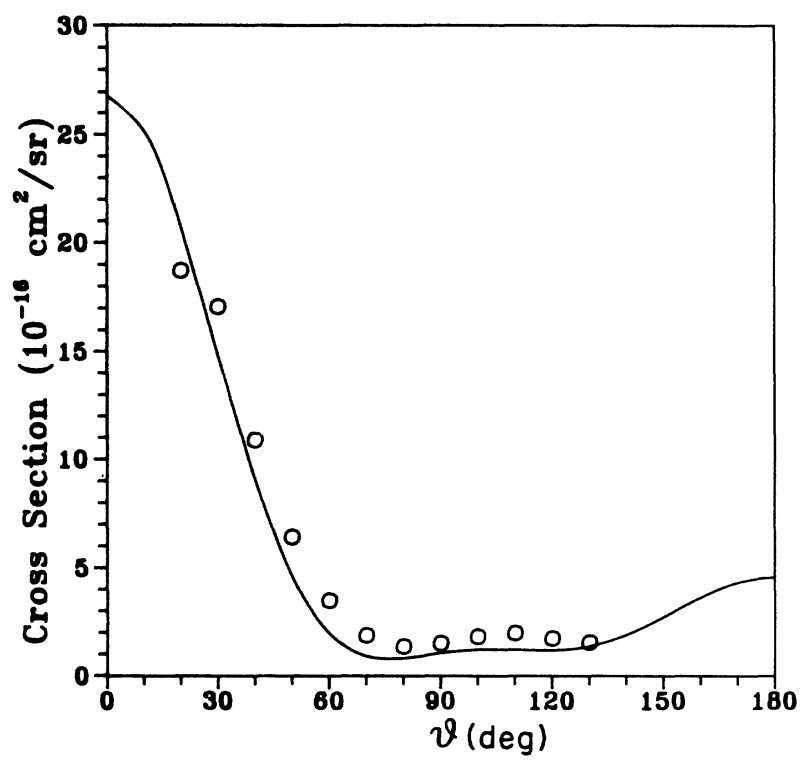

FIG. 5. Same as in Fig. 2, at $7.5 \mathrm{eV}$.

be expected, due to our neglect of polarization. Above 4 $\mathrm{eV}$, however, the comparison between our results and those of Tanaka and co-workers is in general quite satisfactory. Two points are especially worth noting here. First, at 4 and $5 \mathrm{eV}$, we predict significant enhancement of the cross section in the experimentally inaccessible backscattering region. This backward peaking makes a large contribution to the total elastic and especially to the momentum-transfer cross section. The integral and momentum-transfer cross sections reported by Tanaka and co-workers ${ }^{6}$ rely on extrapolation of the differential cross section to $180^{\circ}$. At these energies the differential

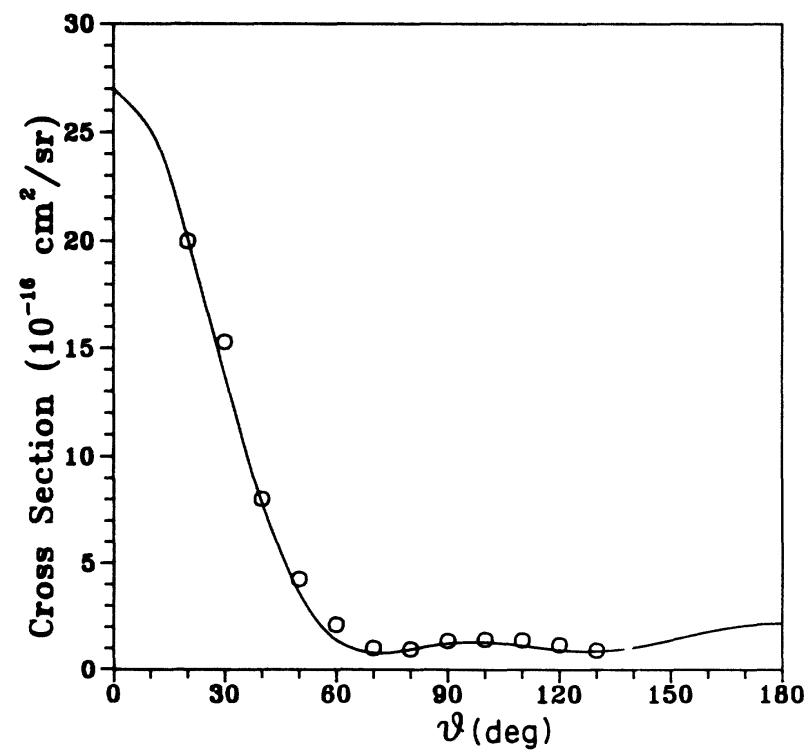

FIG. 6. Same as in Fig. 2, at $10 \mathrm{eV}$. 


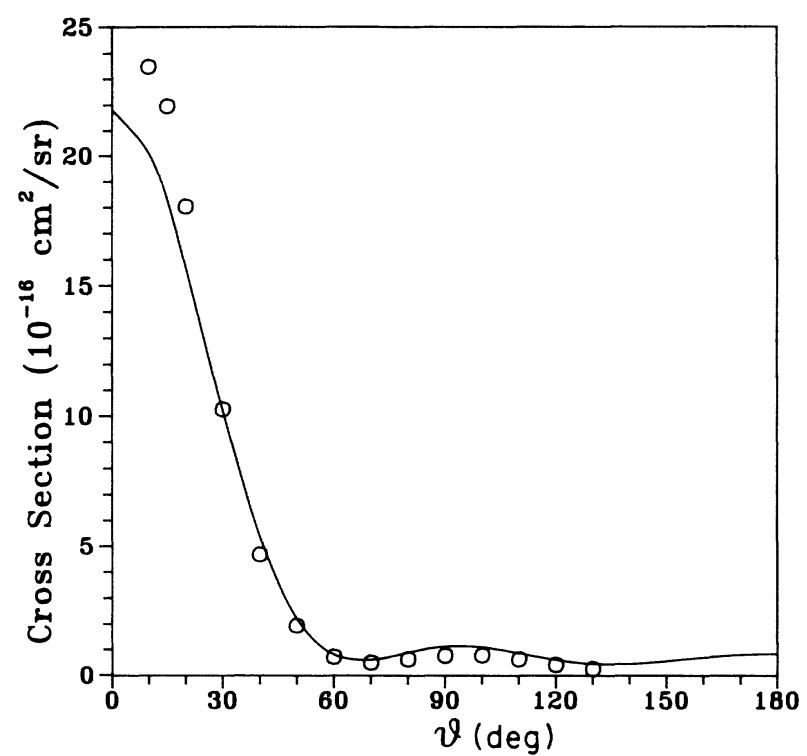

FIG. 7. Same as in Fig. 2, at $15 \mathrm{eV}$.

cross sections are quite flat up to the last measured point at $130^{\circ}$. As a result, their integrated values are almost certainly too low in this energy range, shifting the apparent peak in the total and momentum-transfer cross sections to higher energies. The backward peaking revealed in the present calculation, which is quite similar to that seen in $\mathrm{CH}_{4},{ }^{21,22}$ thus accounts for at least part of the disagreement between the total cross section of Ref. 6 and those of Refs. 5 and 7 . Consistent with this observation is the improved agreement of the experimental results at somewhat higher energies, where our calculation shows a rapid decline in the backward peaking.

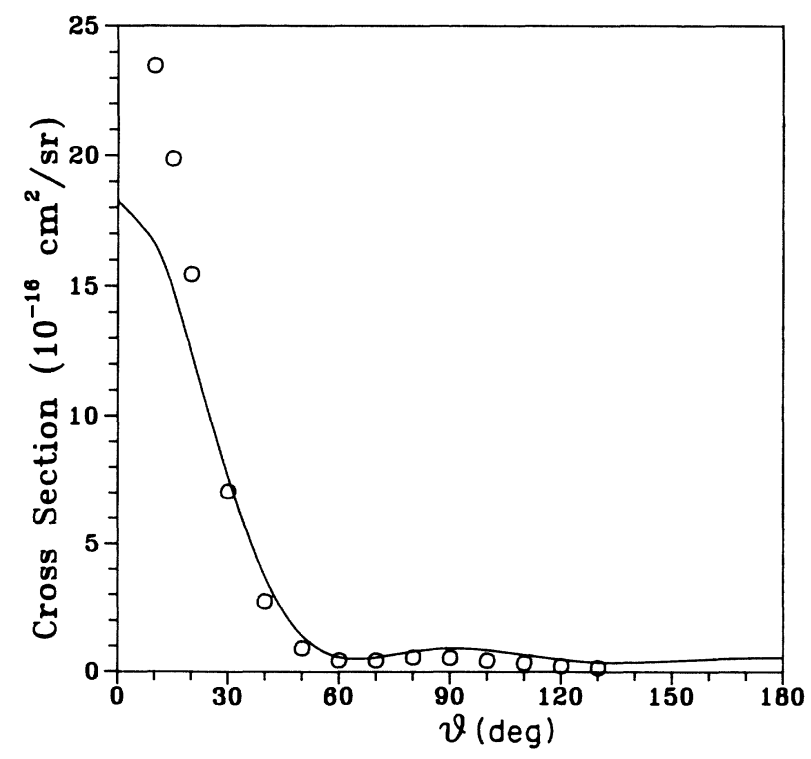

FIG. 8. Same as in Fig. 2, at $20 \mathrm{eV}$.

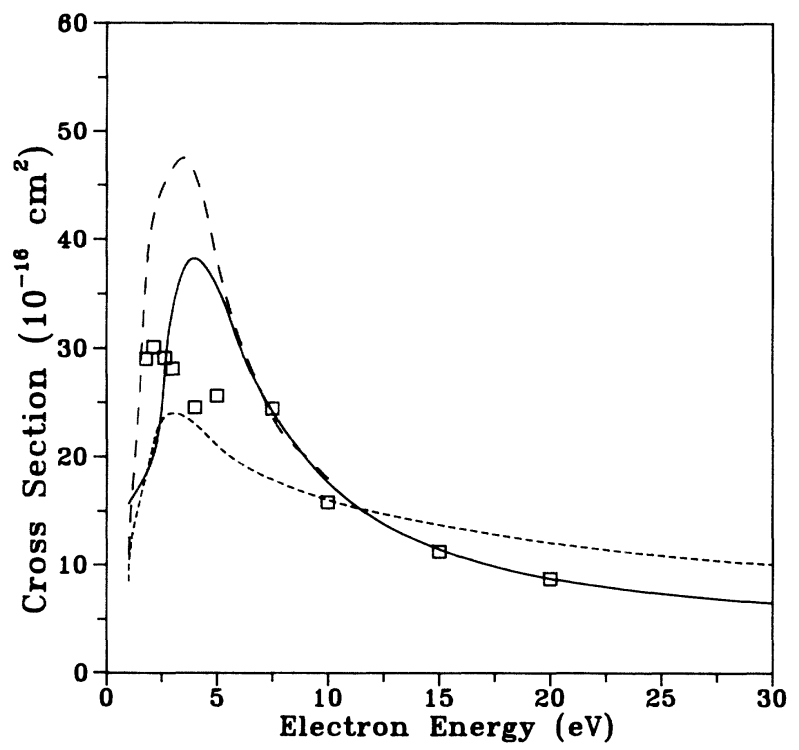

FIG. 9. Momentum-transfer cross section for $e^{-}-\mathrm{SiH}_{4}$ collisions. Calculated values shown are this work (solid line), results of Ref. 14 (long dashes), and the derived values of Ref. 9 (short dashes); the squares are the experimental measurements of Ref. 6.

The second point to be noted from Figs. $2-8$ is that our results at angles smaller than $20^{\circ}$ begin to fall below those of Tanaka and co-workers at higher energies. Although experimental error bars are not indicated in Ref. 6 , we attribute this discrepancy in part to the growing importance of higher partial waves, which are not adequately represented in the fairly modest $L^{2}$ expansion of the wave function which we employ. Adequate representation of these contributions would require additional diffuse Gaussians and Gaussians of higher symmetry type, such as $f$ functions on $\mathrm{Si}$ or $p$ functions on $\mathrm{H}$. On the other hand, the near-forward scattering makes only a small contribution to the momentum-transfer and total elastic cross sections, and the agreement of our results for those quantities with the values of Ref. 6 is only slightly affected.

Figure 9 shows our momentum-transfer cross section, along with the experimental values of Tanaka and coworkers, ${ }^{6}$ the theoretical result of Jain and Thomspon, ${ }^{14}$ and the derived values of Ohmori, Shimozuma, and Tagashira. ${ }^{9}$ As would be expected from the preceding discussion of the differential cross section, our calculation reproduces well the measurements of Ref. 6, except at the lower energies, where the extrapolation used in Ref. 6 probably underestimates the backward peaking. The calculation of Ref. 14 appears to overestimate the peak in the momentum-transfer cross section; however, at higher energies the agreement with the present result is quite good. The estimate of Ohmori, Shimozuma, and Tagashira, ${ }^{9}$ derived from fitting the results of calculations to swarm data, is reasonably close to the data of Ref. 6; however, our results suggest that the peak value of ap- 
proximately $24 \times 10^{-16} \mathrm{~cm}^{2}$ reported in Ref. 9 is somewhat too low.

\section{DISCUSSION}

We have reported static-exchange results for lowenergy electron-silane scattering. Except at the lowest energies, where polarization cannot be neglected, our calculation agrees rather well with available experiments. Indeed, the agreement is perhaps better than could have been anticipated for such a polarizable molecule. These results are highly encouraging, insofar as they suggest that static-exchange results may be useful in describing the scattering of electrons with energies on the order of $10 \mathrm{eV}$ by still larger molecules. In fact, studies now under way for molecules such as disilane $\left(\mathrm{Si}_{2} \mathrm{H}_{6}\right)$ show similar behavior. Earlier $e^{-}-\mathrm{SiH}_{4}$ calculations ${ }^{12-18}$ based on various model potentials have yielded larger values overall than the present results; in contrast, modelpotential $^{33-35}$ and static-exchange ${ }^{21,36,37}$ calculations agreed quite well for $e^{-}-\mathrm{CH}_{4}$ scattering. The reasons for this difference are not yet clear.

The present work illustrates the usefulness of theoretical studies in elucidating discrepancies between experimental results obtained by different techniques. In particular, we have seen that, where backscattering is important, extrapolation of the differential cross section from measurements at intermediate angles may lead to underestimation of the total and momentum-transfer cross sections, and so may change the apparent positions of the resonance maxima. Calculations can provide the experi- mentally inaccessible high-angle scattering data and thus identify this source of disagreement.

In summary, we have found the SMC procedure to yield very satisfactory elastic-scattering results for silane over the range of approximately $4-30 \mathrm{eV}$. Further study of this technologically and theoretically interesting molecule is certainly warranted. In the future, we plan to investigate the lower-energy scattering with polarization effects included and to begin to explore the vibrational and electronic excitation processes, for which experimental data ${ }^{6,11}$ are still quite scarce.

\section{ACKNOWLEDGMENTS}

This work was supported by the National Science Foundation under Grant No. PHY-8604242, the Innovative Science and Technology Program of the Strategic Defense Initiative Organization under Contract No. DAAL03-86-K-0140 of the U.S. Army Research Office, and NASA-Ames Cooperative Agreement No. NCC2319. We thank Professor John Moore and Dr. David Spence for providing their experimental results in advance of publication. We also thank Professor O. Sueoka for providing unpublished results. This work made use of facilities provided by the National Center for Supercomputer Applications at the University of Illinois, which is supported by the National Science Foundation, and of facilities provided through the Supercomputing Project of the Jet Propulsion Laboratory, including those of the $\mathrm{Nu}$ merical Aerodynamic Simulation Program at the NASA-Ames Research Center.
${ }^{1}$ W. E. Spear, Adv. Phys. 26, 811 (1977).

${ }^{2}$ W. J. Pollock, Trans. Faraday Soc. 64, 2919 (1968).

${ }^{3}$ J. C. Giordan, J. Am. Chem. Soc. 105, 6544 (1983).

${ }^{4}$ S. Mori, Y. Katayama, and O. Sueoka, At. Coll. Res. Jpn. 11, 19 (1985).

${ }^{5} \mathrm{O}$. Sueoka (private communication).

${ }^{6}$ H. Tanaka, L. Boesten, H. Sato, M. Kimura, M. A. Dillon, and D. Spence, J. Phys. B 23, 577 (1990).

${ }^{7}$ H.-X. Wan, J. H. Moore, and J. A. Tossell, J. Chem. Phys. 91, 7340 (1989).

${ }^{8}$ M. Hayashi (unpublished).

${ }^{9}$ Y. Ohmori, M. Shimozuma, and H. Tagashira, J. Phys. D 19, 1029 (1986).

${ }^{10}$ A. Garscadden, G. L. Duke, and W. F. Bailey, Appl. Phys. Lett. 43, 1012 (1983).

${ }^{11}$ M. Tronc, A. Hitchcock, and F. Edard, J. Phys. B 22, L207 (1989).

${ }^{12}$ J. A. Tossell and J. W. Davenport, J. Chem. Phys. 80, 813 (1984).

${ }^{13}$ A. Jain, J. Chem. Phys. 86, 1289 (1987).

${ }^{14}$ A. Jain and D. G. Thompson, J. Phys. B 20, 2861 (1987).

${ }^{15}$ A. K. Jain, A. N. Tripathi, and A. Jain, J. Phys. B 20, L389 (1987).

${ }^{16}$ F. A. Gianturco, L. C. Pantano, and S. Scialla, Phys. Rev. A 36, 557 (1987).
${ }^{17}$ J. Yuan, J. Phys. B 21, 2737 (1988).

18J. Yuan, J. Phys. B 22, 2589 (1989).

${ }^{19}$ K. Takatsuka and V. McKoy, Phys. Rev. A 24, 2473 (1981).

${ }^{20}$ M. A. P. Lima and V. McKoy, Phys. Rev. A 38, 501 (1988).

${ }^{21}$ M. A. P. Lima, T. L. Gibson, W. M. Huo, and V. McKoy, Phys. Rev. A 32, 2696 (1985).

${ }^{22}$ M. A. P. Lima, K. Watari, and V. McKoy, Phys. Rev. A 39, 4312 (1989).

${ }^{23}$ T. L. Gibson, M. A. P. Lima, K. Takatsuka, and V. McKoy, Phys. Rev. A 30, 3005 (1984).

${ }^{24}$ W. M. Huo, T. L. Gibson, M. A. P. Lima, and V. McKoy, Phys. Rev. A 36, 1632 (1987).

${ }^{25}$ W. M. Huo, M. A. P. Lima, T. L. Gibson, and V. McKoy, Phys. Rev. A 36, 1642 (1987).

${ }^{26}$ L. M. Brescansin, M. A. P. Lima, T. L. Gibson, and V. McKoy, J. Chem. Phys. 85, 1854 (1986).

${ }^{27}$ H. P. Pritchard, M. A. P. Lima, and V. McKoy, Phys. Rev. A 39, 2392 (1989).

${ }^{28}$ A. D. McClean and G. S. Chandler, J. Chem. Phys. 72, 5639 (1980).

${ }^{29}$ J. Pacansky and M. Dupuis, J. Chem. Phys. 68, 4277 (1978); R. Poirier, R. Kari, and I. G. Csizmadia, Handbook of Gaussian Basis Sets (Elsevier, Amsterdam, 1985), p. 158.

${ }^{30}$ F. B. Van Duijneveldt (unpublished).

${ }^{31}$ M. A. P. Lima, L. M. Brescansin, A. J. R. da Silva, C. Win- 
stead, and V. McKoy, Phys. Rev. A 41, 327 (1990).

${ }^{32}$ M. A. P. Lima, C. Winstead, and V. McKoy (unpublished).

${ }^{33}$ F. A. Gianturco and D. G. Thompson, J. Phys. B 13, 613 (1980).

${ }^{34}$ A. Jain and D. G. Thompson, J. Phys. B 15, L631 (1982).
${ }^{35}$ A. Jain, J. Chem. Phys. 81, 724 (1984).

${ }^{36}$ P. McNaughten and D. G. Thompson, J. Phys. B 21, L703 (1988).

${ }^{37}$ C. W. McCurdy and T. N. Rescigno, Phys. Rev. A 39, 4487 (1989). 